\title{
Parque Estadual de Campos do Jordão: Avaliação Quantitativa de Visitação
}

\author{
Alexandre Schiavetti \\ Maria de Jesus Robim² \\ Maria Eugênia Bruck de Moraes ${ }^{3}$
}

RESUMO: Como um dos instrumentos disponíveis para a verificação dos níveis de consecução dos objetivos propostos nos programas de uso público das unidades de conservação, procedeu-se a avaliação quantitativa da visitação no Parque Estadual de Campos do Jordão (PECJ), São Paulo, através da coleta de dados e informações referentes aoperíodo de dezanos, entre 1985 e 1994.

PALAVRAS-CHAVE: Turismo e conservação de áreas naturais; Parque Estadual deCampos do Jordão; visitação pública; unidades de conservação.

ABSTRACT: Like one of the available tools to verification of the altainment of the objectives proposal in the using public program of the conservation units, we proceded the quantify estimate of the visitors in the State Park of Camiposdo Jordão (PECJ), SãoPaulo, through collecting data and informations conceming to a tenyears, between 1985 and 1994.

KEY WORDS: Tourism and conservation of areas; Campos doJordãoState Park; public visitation; unity of conservation.

1. Ecólogo. Mestre em Ciências da Engenharia Ambiental pela USP - São Carlos (SP).

End. para corresp.: Av. 9 de Julho, 1227 - Centro - 13560-590 - São Carlos - SP - Brasil.

2. Bióloga. Pesquisadora Científica no Instituto Florestal - SP. Doutoranda em Ecologia e Recursos Naturais pela Universidade Federal de São Carlos.

3. Ecóloga. Mestranda em Ciências da Engenharia Ambiental pela USP - São Carlos. 


\section{Introduçāo}

Segundo Boo (1990) o ecoturismo fundamenta-se sobre dois princípios básicos: o primeiro é que a proteção dos recursos naturais das áreas visitadas não poderá ser comprometida; o segundo é que as populações vizinhas a estas áreas devem ser envolvidas e beneficiadas pelo ecoturismo.

Budowsky (1976) sugere que há três diferentes relações que podem existir entre a conservação e o turismo:

- conflito: em que a presença do turismo é prejudicial à conservação;

- coexistência: em que há pouco contato entre turismo e os interesses de conservação;

- simbiose: em que ambos interesses, do turismo e da conservação, derivam benefícios para a relação.

As categorias de Unidades de Conservação (UC) que em decorrência de seu enquadramento aos objetivos básicos do manejo, contemplam o desenvolvimento de atividades turísticas no Brasil, são:

- parques;

- monumentos naturais;

- reservas de fauna;

- áreas de proteção ambiental;

- florestas nacionais

As Áreas Especiais de Interesses Turisticos, previstas pela Embratur, destinam-se justamente a priorizar o desenvolvimento do turismo em áreas con reconhecida vocação natural para essa atividade, podendo ser compartilhada de forma equilibrada com outras formas de ocupação do território (Pires, 1993). Umadas poucas áreas implantadas com esta forma de manejo é a Área Especial de Interesse Turístico do Manumbi(PR).

Nelson (1994) discute avariedade de termos existentes para definir o turismo em áreas naturais, bem como seus principios e caracteristicas.

A visitação pública em algumas Unịdades Estaduais Paulistas é o alvo das atenções e está baseada nas diretrizes indicadas por Cervantes etal. (1992), nas quais

o programa de Uso Público do Instituto Florestal tem porobjetivo propiciar lazer, recreação e educação ambiental à comunidade, bem como despertar uma consciência critica para a necessidade de conservaçāo dos recursos naturais nas Unidades de Conservaçāo sob sua administração.

Nas Unidades de Conservação têm-se trabalhado o ecoturismo numa aproximação muito forte com a educação ambiental (Furlan, 1996) ou com a interpretação ambiental (Pagani et al., 1996), das quais muitas estratégias têm sido adaptadas para o programa de ecoturismo.

Oecoturismo, como alternativa econômica para região, na qual está inserida uma UC, pode possibilitar a sua entrada no desenvolvimento regional, visto a forte atração exercida por estas áreas no público em geral, porém deve-se levar em conta a forte dependência, criada pela comunidade vizinha, da atividade turística, a qual possui sazonalidades.

Nessas áreas ocorre ampla variação sazonal na sua visitação, o que implica em grandes concentrações humanas em determinadas épocas. Osemeobo (1989) identificou os problemas deáreas verdes urbanas ezoológicas da Nigéria, mostrando que a sazonalidade do público, principalmente em épocas de férias, implica grandes impactos para as áreas estudadas.

Magroet al. (1990), trabalhando em um Parque Estadual paulista, mostraram que a variação da visitação deve-se à freqüiência de público às áreas vizinlıas, no caso uma estância balneária que tem elevação do número de visitantes na temporada de verão. Devido às grandes concentrações em épocas determinadas, a tendência nessas áreas é a abertura de novos locais para a recreação, propiciando diferentes experiências recreativas, visando a espacialização dos visitantes (Morris \& Dickinson, 1987; Nelson, 1994). Porém, após algunsanos, há estagnação natural do número de visitantes, o que pode gerar uma estagnação também na economia local.

O aumento do número de turistas, ou a implantação de planos de desenvolvimento turísticos, não deve ser uma estratégia para todas as UCs; somente certas áreas têm infra-estrutura, facilidades de transporte e hotéis para suportar esta indústria. Em primeiro plano, beneficios do turismo podem ser evidentes em nive nacional, porém padrões culturais e econômicos locais podem ser seriamente modificados (Budowsky, 1977).

Estudos especificos, como o perfil dos visitantes (qualitativo e quantitativo), expectativas, padrões de visitação e seus impactos na área, são elementos essenciais para a elaboração de um plano para o manejo da visitação em áreas protegidas(Cebbalos-Lascuráin, 1996).

Bini et.al.(1992), analisando a visitação em dois Parques Nacionais, indicam a "urgência na atenção que deve ser dada aos planos de visitação dos parques" e indicam maior número de sugestões voltadas para a infra-estrutura.

\section{Área de Estudo}

Objeto de intensa demanda turística, o município de Campos do Jordão, anteriormente apenas conhecido pelas suas boas qualidades climáticas, favoráveis ao tratamento de doenças pulmonares, apresenta hojeelevado crescimento, combase principal no comércio de malhas e hotelaria, sendo que o turismo encontra atrativos nas belezas cênicas das paisagens montanas, onde se destaca o componente vegetal; e no clima tropical de altitude, com $14^{\circ} \mathrm{C}$ de temperatura média anual. 
As características turísticas da cidade levaram-na a um crescimento populacional anormal no que se refere a sua forma, deslocando pessoas de fora, ora em caráter temporário, ora permanente, provocando um contingente de pessoas acima do potencial residente da cidade (Paulo Filho, 1986).

Quanto às características ambientais de Campos do Jordão a vegetação se distribui em dois grandes tipos: a mata e a vegetação campestre. De um modo geral apresentam-se profundamente modificadas pela influência do homem. Do ponto de vista paisagístico, sobressai a presença da floresta de Araucaria-Podocarpus, dentre as formações naturais, e de extensos reflorestamentos de coniferas dentre as antropogênicas (Schiavetti, 1995). Seus rios mais importantes, o Sapucaí e o Capivari atravessam a área urbana, estando comprometidos quanto a sua qualidade de água. A área de captação de água para o município se encontra em um Parque Estadual, o Parque Estadual dos Mananciais de Campos do Jordão (Schiavetti, 1997)

Criadoem 27 de março de 1941, o ParqueEstadual deCamposdo Jordão, com 8.341 hectares, compreende aproximadamente um terço do municipio da estância Climática que se localiza na faceocidental dasencostas da Serra daMantiqueira, junto à fronteira entre São Paulo e Minas Gerais.

Em 1974 especialistas alemães, junto com pesquisadores do Instituto Florestal, realizaram estudossobrea paisagem do PECJ, culminando em $1975 \mathrm{com}$ o Plano de Manejo do Parque Estadual de Campos do Jordão (Seibert, et al. 1975), o primeiro plano de manejo realizado no Brasil. Atualmente há a necessidade emergencial de sua revisão, devido às modificações na área do parque, ao aumento da visi tação e aos novos conhecimentos na área de manejo de Unidades de Conservação.

\section{Metodologia}

A obtenção dos resultados, relativos ao campo quantitativo, baseou-se na análise de documentos encontrados nos arquivos do Instituto Florestal, dentre os quais se destacam os processos SMA $n^{\circ} 42385 / 80$ e $n^{\circ} 41771 / 91$, em entrevistas com funcionários responsáveis pelas atividades de uso público, além de observações diretas.

Os dados analisados se referem ao período de dez anos, de 1985 a 1994, e a confiabilidade dos registros foi verificada pela checagem dos volumes referentes a períodos aleatoriamente escollidos, estando estas fontes disponíveis no escritório do Parque Estadual.

Mais recentemente, elementos diversos foram colecionados, o que possibilitou conhecer a estrutura da visitação ora quantificada, através da análise de componentes, como faixa etária e procedência, correlacionados com os fatores externos e internos à área natural protegida (Moraes \& Schiavetti, 1997).
TABELA 1 - NÜMERO DE VISITANTES POR MÊS NO PERIODO DE 1985 - 1994

\begin{tabular}{|c|c|c|c|c|c|c|c|c|c|c|}
\hline Mos/Ano & 1985 & 1986 & 1987 & 1988 & 1989 & 1990: & 1991 & 1992 & 1992 & 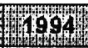 \\
\hline Janelio & 9723 & 12486 & 8847 & 14223 & 12375 & 15899 & 7297 & 8391 & 7778 & 11980 \\
\hline Fevereiro & 10231 & 8245 & 6769 & 11413 & 10780 & 13154 & 5462 & 4765 & 4386 & 9302 \\
\hline Marso & 4993 & 8508 & 10870 & 8132 & 11739 & 6853 & 3770 & 7245 & 3429 & 4119 \\
\hline Abril & 11134 & 13471 & 11625 & 14002 & 18567 & 15410 & 5103 & 9627 & 8110 & 11628 \\
\hline Malo & 8618 & 14976 & 10143 & 14105 & 14776 & 12854 & 11683 & 9157 & 9988 & 8341 \\
\hline Sunho & 19547 & 8385 & 8945 & 10385 & 13666 & 10574 & 8052 & 12428 & 9284 & 13674 \\
\hline Juiho & 7108 & 18399 & 18329 & 25374 & 22573 & 15726 & 17056 & 17595 & 19051 & 21530 \\
\hline Agosto & 8010 & 8010 & 8089 & 8082 & 11313 & 6197 & 8704 & 6492 & 6101 & 9503 \\
\hline Setembro & 8727 & 9877 & 9364 & 11340 & 16247 & 5731 & 7957 & 5430 & 6740 & 8037 \\
\hline Outubro & 12023 & 10540 & 9613 & 13033 & 14409 & 6530 & 5386 & 6079 & 8014 & 8516 \\
\hline Novembro & 8965 & 7768 & 8037 & 7292 & 9305 & 6414 & 5179 & 4748 & 7104 & 6819 \\
\hline Dezembro & 7781 & 5171 & 7742 & 7379 & 6599 & 5907 & 5801 & 5528 & 5348 & 7409 \\
\hline TOTAL & 121390 & 125836 & 118373 & 144760 & 162349 & 121249 & 91450 & 97485 & 95333 & 120858 \\
\hline Variacaso $(\%)$ & 0 & 4 & -6 & 22 & 12 & -25 & -24 & 6 & -2 & 27 \\
\hline
\end{tabular}

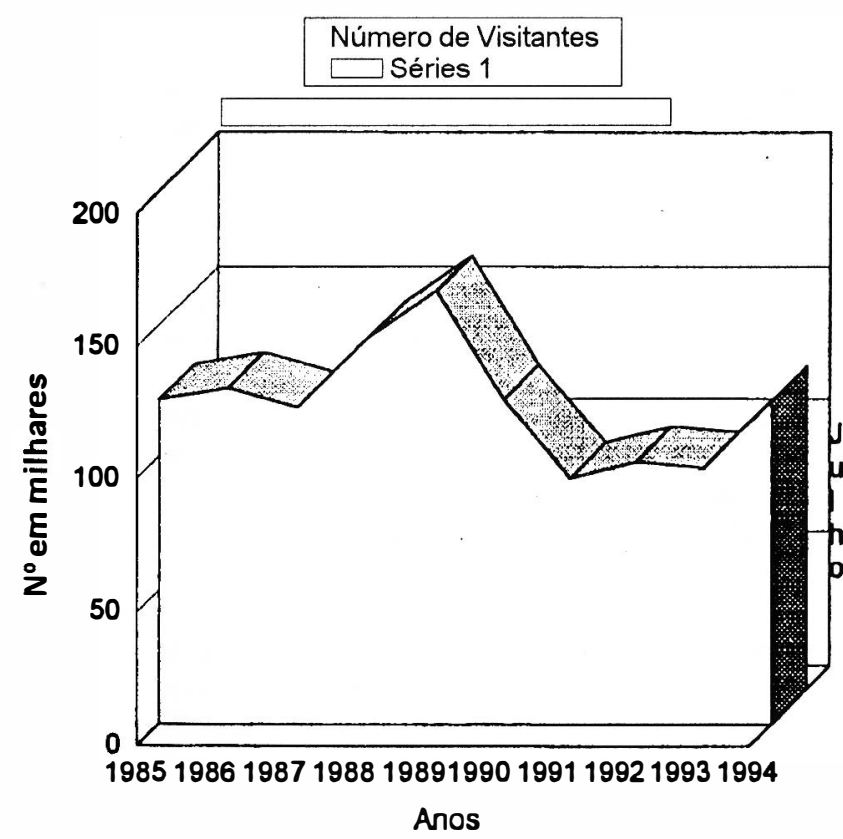

FIGURA 1 - NÚMERO DE VISITANTES NO PARQUE ESTADUAL DE CAMPOS DO JORDÃO, NO PERIODO DE 1985 - 1994 


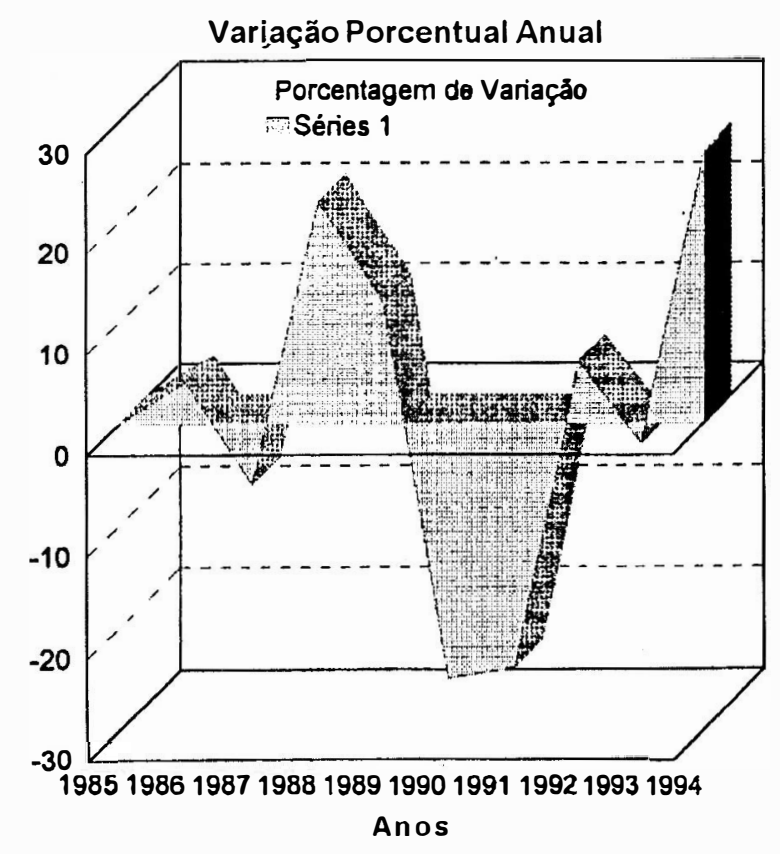

FIGURA 2 - VARIAÇÃO PORCENTUAL DA VISITAÇÃO NO PARQUE ESTADUAL DE CAMPOS DO JORDÃO NO PERIODO DE 1985 - 1994

\section{Resultados e Discussāo}

A Tabela 1 apresenta a quantificação dos visitantes do PECJ, no período de 1985a 1994, distribuídos mensalmente, e a variação existente entre os anos, colocando-se o ano de 1985 como inicial.

As Figuras 1 e 2 mostram a dinâmica desta variação, notando-se na Figura 1 a grande quantidade devisitantes noano de 1989 e salientando-se na Figura 2 a grande variação (negativa) após o ano de 1989.

No periodo estudado foram computados 1.199 .083 visitantes, registrando-se a média de $119.908,3$ porano, com máxima verificadaem 1989e mínimaafluência de públicoem 1993.

De ordem genérica para a Estância de Campos do Jordão, onde a mídia concentra as atenções durante o inverno ${ }^{4}$, lambém se verifica a maior freqüência de visitantes no mês de julho, quando o Parque Estadual, em 1994, recebeu a média de 718 pessoas ao dia.

\footnotetext{
4. Nessa cidade anualmente realiza-se, em julho, o Festival de Inveno, envolvendo atividades e eventos artísticos
} e culturais.
A ocorrência de variações no número de visitantes deve ser considerada como reflexo de diversos fatores, desde situação econômicado períodoaté a mellhoria de infra-estrutura, passando, no caso de uma estância climática, por acontecimentos de ordem meteorológica. Estas características são também discutidas em Osemeobo (1989) e Magri etal., (1990).

No período analisado de dez anos ocorreram diversas mudanças na situação política-econômica brasileira, porém pouco se percebe nos dadosanalisados, provavelmente devido ao público visitante da estância climática de Campos do Jordão pertencer às classes econômicas média e alta.

A variação existente reflete a disponibilidade de se pagar porum recursonatural, poisa grande queda no número de visitantesé observada apóso inícioda taxa de visitação na Unidade (setembro de 1990), mostrando-se com isso a pequena importância dada à conservação dos recursos naturais, ante a disponibilidade aopagamento pelautilização.

Apesar desta característica ter sido observada, estudos como o de Peters et al. (1989) mostram que áreas naturais podem possuir maiorvalor econômico quando em seu estado natural, gerando benefícios diretos e indiretos à comunidade vizinha. Estacaracterística pode scr atribuída também aofluxo turísticopara oParqueEstadual de Campos do Jordão, haja vista o crescente número de estabelecimentos comerciais associados ao turismo construídos ao longo da estrada de acesso a este destino.

Outro acontecimento no qual o reflexo pode ser sentido no número de visitantes nesta Unidade foi a expectativa de neve nos anos de 1988 e 1989, fato pouco comume m terras brasileiras, salientando-se a temperatura múnima de $-4,4^{\circ} \mathrm{C}$, ocorrida em 1988 e repetida em 1994. Acredita-se também que a grandevisitação ocorrida no ano de 1994 deve ser atribuída à estabilidade econômica e ao aumento do nível de renda da população vivenciada pela sociedade brasileira no período.

\section{Conclusāo}

As quantificaçõesapresentadaseanalisadas permitem concluir sobre a importância doandamento de tais levantamentos, num processo de avaliação contínua, cujos resultados podem ser utilizados para o monitoramento dos programas de uso público da própria Unidade de Conservação, assim como para referenciar o planejamento de outras Unidades, ressalvadas as peculiaridades distintas quc, ainda, devem considerar as áreas circunvizinhas, aspectos culturais, demanda e disponibilidade de recursos.

A realização de estudos sobre o perfil dos usuários do Parque, considerando os períodos de concentração de visitantes, demonstrados como nos meses de inverno devem ser mantidos, objetivando-se a identificação permanente das necessidades do visitante, bem como da mudança do perfil do público-alvo.

A avaliação econômica dos benefícios do ecoturismo para a população do entorno e os impactos ambientais do turismo devem ser quantificados, procurandose direcionar os benefícios do ecoturismo para a região.

O Plano de Manejo do PECJ prevê a espacialização dos visitantes nas zonas de uso intensivo e extensivo. Defasado, deve ser revisto e atualizado, envolvendo 
de forma participativa os setores da comunidade, das universidades e dos poderes públicos municipal e estadual, no processo de planejamento.

Assim, no caso do PECJ, a administração deve-se preocupar com a oferta de atrativos (trilhas interpretativas, exposições etc.) e a segurança do público visitante, monitorando e avaliando o atual Programa de Uso Público da Unidade.

\section{Referências Bibliográficas}

BINI, L.M. et al. 1992. Caracterização do pertil dos visitantes dos Parques Nacionais de Aparados da Serra (RS) e Brasilia (DF). In: CONGRESSO NACIONAL SOBRE ESSÊNCIAS NATIVAS, 2, Revista do Instituto Florestal, ed. especial, v.4: 1106-1108.

BOO, E. 1990. Ecolurismo: potenciales y escollos. Washington, D.C.; WWF \& The Conservation Foudation. 226 p. BUDOWSKY, G. 1976. Tourism and envirommental conservation: contlict, coexistence or simbiosis? Environmental Consentation, v. 3, n. 1, p. 27-32.

p. 3-16.

1977. Tourism and environmental conservation: conflict, coexistence or simbiosis? Parks, v. I, n. 4 ,

CEBALLOS-LASCURÁIN, H. 1996. Tourism, ecoturism and protected areas. The state of nature-based tourism around the world and guidelines for its development. IUCN, Gland, Switezerland and Cambridge. UK. XIV. 301p.

CERVANTES, A.L.A. et al. 1992. Diretrizes para os programas de uso púbilico do Instituto Florestal de São Paulo SMA, São Paulo. In: CONGRESSO NAC.IONAL SOBRE ESSÊNC.IAS NATIVAS, 2, Revista do Instituto Florestal, ed. especial, v. 4, p. 1076-80.

FURLAN, S. 1996. Unidades de conservação insular: considerações sobre a dinânica insular, planos de manejo e turismo ambiental. In: DE LEMOS, A.I. (org.) Turismo: imipactos socioambientais. Hucitec. p. 114-36.

GOVERNO DO ESTADO DE SÃO PAULO. SECRETARIA DO MEIO AMBIENTE - INSTITUTO FLORESTAL. 1980. Processo SMLA $n^{\circ}$ 42385/80. Assunto: Encaminhamento mensal de visitantes referentes aos meses de setembro à novenibro de $1980.245 \mathrm{p}$.

GOVERNO DO ESTAIJO DE SÃO PAULO SECRETARIA DOMEIO AMBIENTE - INSTITUTO FLORESTAL. 1991. Processo SMA $n^{\circ}$ 41771/91. Assunto: cobrança de ingresso no Pargue Estadual de Camipos do Jordão. $32 \mathrm{p}$.

MAGRO, T.C.; GRANJA, C.M. \& MENDES, F.B.G. 1990. Características do usuário do Parque Estadual da Ilha Anchieta - subsídios para o plano interpretativo. In: C.ONGRESSO FLORESTAL BRASILEIRO, 6, Anais, p. $766-78$

MORAES, M.E.B. \& SCHIA VETTI, A. 1997. Análise do perfil do visitante e moradores do Parque Estadual de Campos do Jordão: subsidios para un programa de coleta seletiva de lixo. In: SIMPÓSIO DE CIÊNCIAS DA ENGENHA. RIA AMBIENTAL, 2, Anais, p. 167-169.

MORRIS, A. \& DICKINSON, G. 1987. Tourism development in Spain: gowth versus conservation on the Costa Brava. Geography, 72(3/4): 16-25.

NELSON, J.G. 1994. The spread of ecotourism: some planning implications. Environmental Conservation, v. 21 , n. 3 , p. $248-255$.

OSEMEOBO, G.J. 1989. Forest recreational land-use and zoological gardens ina a developing econony: the case of Southem Nigeria. Environmental Consenation, v. 16, n. 1, p. 69-74.

PAGANI, M.I. et al. 1996. As trilhas interpretativas da natureza e o ecoturismo. In: DE LEMOS, A.I. (org.) Turismo: inipactos socioamibientais. Hucitec. p. 151-63.

PAULO FILHO, P. 1986. História de Campos do Jordão. Editora Santuário. 782 p.

PETERS, C.G.; GENTRY, A.H. \& MENDELSOHN, R. 1989. Valuation of a tropical forest in Peruvian Amazonia. Nature, n. 339, p. 655-6.

PIRES, P.S. 1993. Turismo en áreas naturais protegidas. In: Curso sobre Manejo de Áreas Naturais Protegidas. Paraná: Universidade Livre do Meio Ambiente, p. 63-75.

SCHIA VETTI, A. 1995. Campos do JordãoStatePark. In: IEENRY, R. \& SENNA, P.A.C. (org.). XXVI SIL CONGRESS, São Paulo, Mid-congress Excursions, p. 35-6.

1997. Proposta de zoneamento recreativo para o Parque Estadual dos Mananciais de Camipos do Jordão: subsidios para o plano de manejo. São Carlos; EESC-USP. Dissertação de Mestrado. 81 p.

SEIBERT, P. et aI. 1975. Plano de manejo do Parque Estadual de Campos do Jordão. Boletinı Técnico IF. São Paulo, Instituto Florestal. n.19, 153 p.

\section{Recebido em 15/\$/97}

Aprovado em 10/8/97 